\title{
Image Compression Techniques for High Resolution Satellite Imageries using Classical Lifting Scheme
}

\author{
K Nagamani \\ Lecturer, R V College of Engineering \\ Bangalore- 560059
}

\author{
AG Ananth \\ Professor, $R \vee$ College of Engineering \\ Bangalore- 560059.
}

\begin{abstract}
The properties of Wavelet Transform can be successfully applied for analysis and processing of non stationary signals e.g., speech and image processing, data compression and communications. Due to the growing number of applications in various areas, it is necessary to explore the hardware implementation options of the Discrete Wavelet Transform (DWT). The Wavelet Series is just a sampled version of Continuous Wavelet Transform (CWT) and its computation may consume significant amount of time and resources, depending on the required resolution. The Discrete Wavelet Transform (DWT), based on sub-band coding, is a fast computation technique of Wavelet Transform, easy to implement and reduces the computational time and resources. Wavelet Transform uses multi-resolution technique by which different frequencies are analyzed with different resolutions. The study of 2-D DWT architectures reveals that there are two schemes for implementing DWT, one is based on convolution and other is based on lifting scheme. In this paper detailed study of Lifting Scheme has been carried out. Different architectures have been studied and performance parameters such as PSNR and Compression Ratio are determined. After obtaining double precision value of the image of size $256 * 256$ imagery in BMP format, discrete wavelet transforms techniques are applied to obtain the wavelet coefficients for calculating PSNR and Compression ratio. Inverse Discrete wavelet transform are applied to get back the reconstructed image. It is found that for both satellite Rural and Urban imageries, the lifting scheme is very useful for obtaining higher quality of reconstructed images while achieving better PSNR 29 and Compression Ratios $\sim 8$.
\end{abstract}

\section{INTRODUCTION}

The lifting scheme formally introduced by Sweldens ${ }^{1}$ is a wellknown method to create bi-orthogonal wavelet filters from other ones. Usually, a poly-phase decomposition (or lazy wavelet transform, LWT) of the input signal $\mathrm{x} 0$ into sub signals [1]is done, obtaining an approximation signal $\mathrm{x}$ and a detail signal $\mathrm{y}$. Then, lifting steps are performed by predicting the detail signal from the $\mathrm{x}$ samples and updating the approximation signal with the y samples .The so-called prediction $\mathrm{P}$ (or dual lifting) and update U (or primal lifting) steps improve the initial lazy wavelet properties.

$\mathrm{y} 0[\mathrm{n}]=\mathrm{y}[\mathrm{n}]-\mathrm{P}(\mathrm{x}[\mathrm{n}])$

$\mathrm{x} 0[\mathrm{n}]=\mathrm{x}[\mathrm{n}]+\mathrm{U}(\mathrm{y} 0[\mathrm{n}])$

Although every reconstruct able filter bank can be expressed in terms of lifting steps, an explicit decomposition for a family of wavelets is only known for the Cohen-Daubechies-Feauveau wavelet. The lifting algorithm was implemented by writing a Matlab code to analyse and synthesize the image in BMP format. For any given image, the lifting algorithm performs the forward DWT to get the filter coefficients (the analysis step) and encodes the image. The inverse DWT is performed with the same lifting algorithm to obtain back the perfect reconstructed image (synthesis step). The lifting algorithm was applied to 3 test images of $256 * 256$ grey scale test imageries i.e. 'lena. bmp', 'satellite rural.bmp' and 'satellite urban.bmp'. Satellite urban and satellite rural images are significantly used in our research which describes the topology of the area like land, sea and clouds, measure wave heights, sea temperature and elevations, wind speed and direction.

\section{Architecture for lifting based 1D DWT}

The basic principle of the lifting scheme is [1] to factorize the polyphase matrix of a wavelet filter into a sequence of alternating upper and lower triangular matrices and a diagonal matrix. This leads to the wavelet implementation by means of banded-matrix multiplications. Let $\mathrm{h}^{\sim}(\mathrm{z})$ and $\mathrm{g}^{\sim}(\mathrm{z})$ be the low pass and high pass analysis filters, and let $\mathrm{h}(\mathrm{z})$ and $\mathrm{g}(\mathrm{z})$ be the lowpass and highpass synthesis filters. The corresponding polyphase matrices of analysis and synthesis filters are defined as

$$
\begin{aligned}
& P^{\sim}(Z)=\left(\begin{array}{ll}
h_{e}^{\sim}(z) & h_{o}^{\sim}(z) \\
g_{e}^{\sim}(z) & g_{o}^{\sim}(z)
\end{array}\right) \\
& \text { and } \\
& \qquad P(Z)=\left(\begin{array}{ll}
h_{e}(z) & h_{o}(z) \\
g_{e}(z) & g_{o}(z)
\end{array}\right)
\end{aligned}
$$

It has been shown that if $\left[\mathrm{h}^{\sim}, \mathrm{g}^{\sim}\right]$ is a complementary filter pair, then $\mathrm{P}^{\sim}(\mathrm{z})$ can always be factored into lifting steps as

$$
\begin{aligned}
& P_{1}^{\sim}(Z)=\left(\begin{array}{cc}
k_{1} & 0 \\
0 & k_{2}
\end{array}\right) \prod_{1}^{m}\left(\begin{array}{cc}
1 & s_{i}^{\sim}(z) \\
o & 1
\end{array}\right)\left(\begin{array}{cc}
1 & 0 \\
t_{i}^{\sim}(z) & 1
\end{array}\right) \\
& P_{2}^{\sim}(Z)=\left(\begin{array}{cc}
k_{1} & 0 \\
0 & k_{2}
\end{array}\right) \prod_{1}^{m}\left(\begin{array}{cc}
1 & 0 \\
t_{i}^{\sim}(z) & 1
\end{array}\right)\left(\begin{array}{cc}
1 & s_{i}^{\sim}(z) \\
o & 1
\end{array}\right)
\end{aligned}
$$

Where $\mathrm{K}$ is a constant. The two types of lifting schemes are shown in Figure 1.

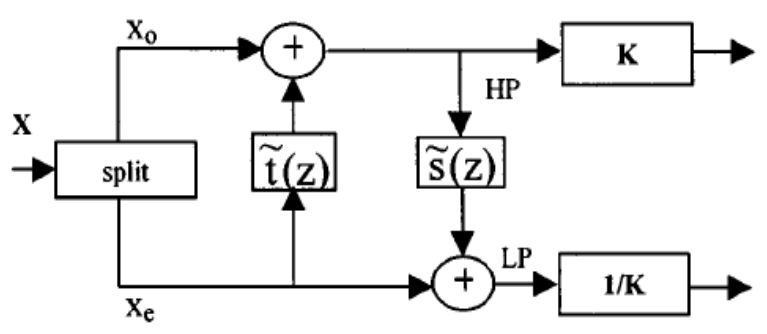

Figure 1.a Lifting schemes scheme 1

Scheme 1 (see Figure 2 (a)), which corresponds to the $\mathrm{P}_{1}^{\sim}(z)$ factorization, consists of three step. 
- Predict step, where the even samples are multiplied by the time domain equivalent of $\mathrm{t}^{\sim}(\mathrm{z})$ and are added to the odd samples.

- Update step, where updated odd samples are multiplied by the time domain equivalent of $\mathrm{s}^{\sim}(\mathrm{z})$ and are added to the even samples.

- $\quad$ Scaling step, where the even samples are multiplied by $1 / \mathrm{K}$ and odd samples by $\mathrm{K}$

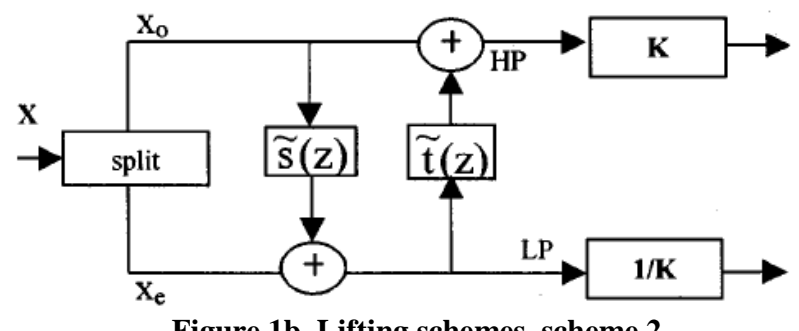

Figure 1b. Lifting schemes scheme 2

In Scheme 2 (see Figure $1 \mathrm{~b}$ ), which corresponds to the $\mathrm{P}_{2}^{\sim}(z)$ factorization, the odd samples are calculated in the first step, and the even samples are calculated in the second step.

It allows a faster implementation of the wavelet transform Traditionally, the fast wavelet transform is calculated with a twoband sub-band transform scheme. The lifting scheme makes optimal use of similarities between the high and low pass filters to speed up the calculation. In some cases the number of operations can be reduced by a factor of two.

\section{ARCHITECTURE FOR 2D DWT}

The de-correlation of an image requires a two-dimensional DWT, which is performed by iterated application of the onedimensional DWT. Viewing the image as [3] a data matrix consisting of rows and columns of signal vectors, a single-level 2-D DWT shall be performed on the image in the following two steps in the following order:

a). The convolution/lifting based 1-d DWT shall be performed on each image row, producing a horizontally low-pass and a horizontally high-pass filtered intermediate data array, each half as wide as the original image array, as illustrated in figure 2(b); b). The convolution/lifting based 1-d DWT shall be applied to each column of both intermediate data arrays to produce four sub-bands as shown in figure 2(c).
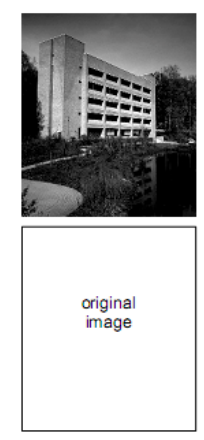

(a)

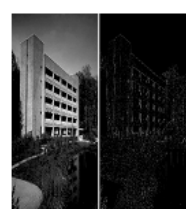

1-d DWT of each
row $\longrightarrow$

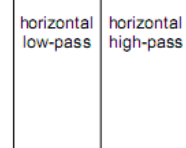

(b)

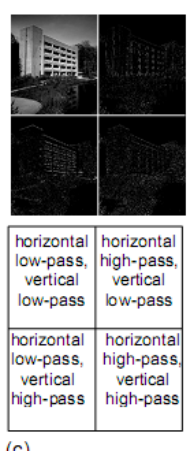

(c)
Figure 2. One -Level 2-D DWT Decomposition of an Image

Each of the four sub-band data arrays obtained is half as wide and half as tall as the original image array.
In Convolution based 2D DWT, the separable 2-D DWT can be implemented by two-channel decomposition of 1-D DWT in the horizontal (row) and vertical (column) dimensions. In stage 1. The 1-D DWT is computed along the rows of the input array to generate the $\mathrm{H}$ high pass) and the $\mathrm{L}$ (low pass) outputs. If the image is of size $\mathrm{N} \times \mathrm{N}$, then the $\mathrm{H}$ and $\mathrm{L}$ output arrays are each of size $\quad \mathrm{N} \times \mathrm{N} / 2$. The $\mathrm{HH}$ and HL output arrays are obtained by computing 1-D DWT along the columns of the $\mathrm{H}$ array. Similarly, the LH and LL output arrays are obtained by computing 1-D DWT along the columns of the $\mathrm{L}$ array. The $\mathrm{HH}$, HL, LH and LL arrays are each of size N/2 x N/2. The LL outputs are sent to Stage 2. The four outputs of Stage 2, namely LLHH, LLHL, LLLH and LLLL, are each of size N/4 x N/4. Out of these, the LLLL outputs are sent to next stage. Thus $\mathrm{N}^{2}$ outputs are generated in stage $1, \mathrm{~N}^{2} / 4$ in stage 2 and $\mathrm{N}^{2} / 2^{\mathrm{m}}$ in stage $\mathrm{m}$. Similarly lifting based 2D DWT can be obtained; using lifting based 1D DWT instead of convolution.

\section{COMPARISON WITH CONVOLUTION}

The number of computations required for calculation of a high pass, low pass pair of wavelet transforms using convolution and lifting scheme is given in Table 1 . The reduction in the number of multiplications for the lifting scheme is significant for odd-tap filters compared with convolution. For even-tap filters, the convolution scheme has fewer or an equal number of multiplications. The number of additions is lower for lifting in both odd and even tap filters. Such reduction in the computational complexity makes lifting-based schemes attractive for both high throughput and low-power applications.

Table 1 showing computational complexity comparison between convolutions and lifting scheme.

\begin{tabular}{|l|l|l|l|l|}
\hline Filters & \multicolumn{2}{|l|}{$\begin{array}{l}\text { Multiplications } \\
\text { /shifts }\end{array}$} & \multicolumn{2}{l|}{ Additions } \\
\hline & Convolution & Lifting & Convolution & Lifting \\
\hline$(5,3)$ & 4 & 2 & 6 & 4 \\
\hline$(9,7)$ & 9 & 5 & 14 & 8 \\
\hline$(2,10)$ & 3 & 4 & 10 & 6 \\
\hline$(6,10)$ & 8 & 8 & 14 & 8 \\
\hline
\end{tabular}

It is clear from the above table that the hardware requirements are less for the lifting scheme compared to convolution method, which finally leads to reduction in computational complexity. Such reduction in the computational complexity makes liftingbased schemes attractive for both high throughput and low power applications.

\section{RESULTS AND DISCUSSION}

The lifting algorithm was implemented by writing a Matlab code to analyze and synthesize the image in BMP format. For a given image, the lifting algorithm performs the forward DWT to get the filter coefficients (the analysis step) and encodes the image. The inverse DWT is performed with the same lifting algorithm to get back the perfect reconstructed image (synthesis step). The lifting scheme is applied to 3 test images. The results obtained are studied for all the input images used in BMP format, i.e. portable grey map. The image sizes are considered to be $256 * 256$ pixels.

The results are as shown in the bar graph Figure 3 as well as in the Table 2 


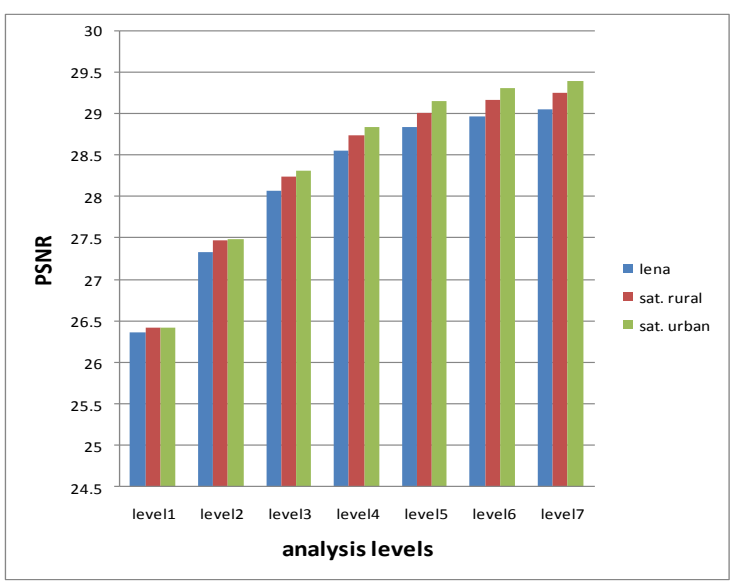

Figure 3. Plot of PSNR v/s level for the three images under study

Peak Signal to Noise Ratio (PSNR): The PSNR is calculated with the following formula

$$
\text { PSNR }=10 \log _{10}\left[\frac{\text { Maxgrey Level } * M N}{\sum_{x y} \mathrm{~g}(\mathrm{xy})-\mathrm{f}(\mathrm{xy})}\right]
$$

Where $\mathrm{g}(\mathrm{x}, \mathrm{y})$ is the compressed image, $\mathrm{f}(\mathrm{x}, \mathrm{y})$ is the raw image $\mathrm{M}$ is the image width, $\mathrm{N}$ is the image height and max. gray level is the max. value of $f(x, y)$. A Max grey level $=255$ has been used (as there are 0 to 255 grey levels represented with 8 bits in the BMP format images).

Table 2 Lifting algorithm results for different images under study

\begin{tabular}{|c|c|c|c|}
\hline Image & Level & $\begin{array}{l}\text { Compression } \\
\text { ratio }\end{array}$ & Psnr \\
\hline \multirow{6}{*}{ LENA } & 1 & 8 & 26.3672 \\
\hline & 2 & 8 & 27.3388 \\
\hline & 3 & 8 & 28.0779 \\
\hline & 4 & 8 & 28.5664 \\
\hline & 5 & 8 & 28.8505 \\
\hline & 6 & 8 & 28.9783 \\
\hline \multirow{6}{*}{$\begin{array}{l}\text { SATELLITE } \\
\text { URBAN }\end{array}$} & 1 & 8 & 26.4170 \\
\hline & 2 & 8 & 27.4908 \\
\hline & 3 & 8 & 28.3176 \\
\hline & 4 & 8 & 28.8490 \\
\hline & 5 & 8 & 29.1629 \\
\hline & 6 & 8 & 29.3222 \\
\hline \multirow{6}{*}{$\begin{array}{l}\text { SATELLITE } \\
\text { RURAL }\end{array}$} & 1 & 8 & 26.4255 \\
\hline & 2 & 8 & 27.4734 \\
\hline & 3 & 8 & 28.2423 \\
\hline & 4 & 8 & 28.7420 \\
\hline & 5 & 8 & 29.0126 \\
\hline & 6 & 8 & 29.1715 \\
\hline
\end{tabular}

It is observed from the Table 2 that, for Lena Image the saturation values are achieved after $3^{\text {rd }}$ level where as for the Satellite Rural and Urban imageries the saturation of PSNR values occurs only after the $5^{\text {th }}$ level. This indicates that by increasing the level of decomposition one can achieve higher PSNR values for satellite imageries where as for standard Lena Image the increase in level of decomposition will not further improve the PSNR values and hence the quality of the reconstructed image. The higher PSNR values achievable for satellite imageries $\sim 29$ at the $5^{\text {th }}$ level indicates that the quality of satellite can be improved with higher levels of decomposition and using lifting schemes. The compression ratio $\sim 8$ remains constant for both type of images which is the maximum compression achievable for Lifting schemes.

\section{CONCLUSIONS}

From the above presentation of results and discussions we can conclude that

1. Lifting schemes can be adopted for the compression of both satellite Rural and Urban imageries for achieving significant compression rations $\sim 8$

2. The satellite imageries for both Rural and Urban scenes indicate that using the lifting schemes one can achieve higher PSNR values 29 with increase in the levels of decomposition..

3. Using the lifting scheme techniques, one can achieve high quality of reconstructed images for the same compression ratio for both satellite Rural and Urban Imageries.

\section{ACKNOWLEDGEMENT}

The Authors are thankful to Dr Ganesh Raj Dy Director, ISRO for providing the satellite images and Head, Dept of Telecommunication, RV Engineering college for encouragement in carrying out the present research work.

\section{REFERENCES}

[1] Swelden, "A Fast Progressive Image Sampling UsingLifting Scheme And Non-Uniform B-Splines", ISIE 2007. IEEE International Symposium on Industrial Electronics. 4-7 June 2007.

[2] Wavelets and Filter Banks by Gilbert Strang and Truong Nguyen, Wellesley-Cambridge Press, 1997

[3] Ripples in Mathematics: the Discrete Wavelet Transform by Arne Jense and Anders la Cour-Harbo, Springer, 2001

[4] 4. Colm Mulcahy, "Image Compression Using The Haar Wavelet Transform", Spelman College Science \& Mathematics Journal, Vol 1, No 1, April 1997, 22-31.

[5] M L Hilton, B D Jawerth and Ayan Sengupta, "Compressing still and moving images with wavelets" published in the journal "Multimedia Systems" vol-2, no-3, 1994.

[6] P J Burt and E H Adelson "The Laplacian Pyramid as a compact image code" IEEE Trans. On Communication. Vol 31, pp 532-540, (1983)

[7] Bryan Usevitch, "A Tutorial on Modern Lossy Wavelet Image Compression : Foundations of JPEG 2000," IEEE Signal Processing Magazine, 2001.

[8] R. A. DeVore, B. Jawerth, and B. J. Lucier, "Image Compression Through Wavelet Transform Coding," IEEE Trans. On Information Theory, Vol. 38, No. 2, pp. 719 746, (1992). 
[9] A. S. Lewis and G. Knowles, "Image Compression Using the 2-D Wavelet Transform", IEEE Trans. on Image Processing, Vol. 1, No. 2, pp. 244-250, April (1992).

[10] J.M.Shapiro, “An Embedded Wavelet Hierarchical Image Coder," Proc. IEEE Int. Conf. Acoust. Speech, Signal Processing, San Francisco, CA, (1992).

[11] J.M.Shapiro,"Embedded Image Coding Using Zerotrees of Wavelet Coefficients", IEEE Trans. on Signal Processing, Vol. 41, pp 3445-3462, (1993)

[12] 12. M Vetterli, J Kovacevic and D J Legall “Perfect Reconstruction Filter Banks for HD TV representation and coding" Image communication Vol 2 essing Magazine . Vol 8, pp 349-364 (1990)

[13] 13. A. Said and W.A. Pearlman, "A New, Fast and Efficient Image Codec Based on Set Partitioning in Hierarchical Trees", IEEE Trans. on Circ and Syst for Video Tech, Vol 6 , no. 3, pp 243-250, June 1996.

[14] 14. Said and A. Pearlman, "An Image Multiresolution Representation for Losssless and Lossy Compression." IEEE Trans. Image Processing, Vol. 5, No. 9, pp 243-250, Sept. 1996.

\section{AUTHORS PROFILE}

Nagamani.K was born on 30/05/1973 in Bangalore, India. She received B.E degree in 1996 from Bangalore University and M.Tech degree in 2006 from Visveswaraih Technical University, Belgaum, India.She is pursuing $\mathrm{PhD}$ Degree. Presently working as Senior Lecturer in Telecommunication at R.V. College of Engineering Bangalore. Her research interests include Digital Compression techniques and Embedded System design.

Dr..A.G. Ananth was born on 3/11/1947, at Bangalore, India. He received M.Sc degree in Nuclear Physics in 1969 from Bangalore University, India. In 1975, the Physical Research Laboratory Ahmadabad awarded him PhD degree in Space physics. He served as Deputy Director at Indian space research organization, Bangalore. Presently working as Professor in Telecommunication Department of R.V College of Engineering, Bangalore. His research interests include Space Applications, Image processing, Image compression, MIMO Systems and Satellite communications. 